\title{
Efficient Algorithms for Concept Space Construction
}

\author{
C.Y. Ng Joseph Lee Felix Cheung Ben Kao David Cheung \\ Department of Computer Science and Information Systems \\ The University of Hong Kong \\ \{cyng, jkwlee, kmcheung, kao, dcheung\}@csis.hku.hk
}

\begin{abstract}
The vocabulary problem in information retrieval arises because authors and indexers often use different terms for the same concept. A thesaurus defines mappings between different but related terms. It is widely used in modern information retrieval systems to solve the vocabulary problem. Chen et al. proposed the concept space approach to automatic thesaurus construction. A concept space contains the associations between every pair of terms. Previous research studies show that concept space is a useful tool for helping information searchers in revising their queries in order to get better results from information retrieval systems. The construction of a concept space, however, is very computationally intensive. In this paper, we propose and evaluate efficient algorithms for constructing concept spaces that include only strong associations. Since weak associations are not useful in thesauri construction, our algorithms use various prunning techniques to avoid computing weak associations to achieve efficiency.
\end{abstract}

Keywords: concept space, thesaurus, information retrieval, text mining

\section{Introduction}

The vocabulary problem has been studied for many years [5,3]. It refers to the failure of a system caused by the variety of terms used by its users during humansystem communication. Furnes et al. studied the tendency of using different terms among different users to describe a similar concept. For example, they discovered that for spontaneous word choice for concepts, in certain domain, the probability that two people choose the same term is less than $20 \%$ [5]. In an information retrieval system, if the keywords that a user specifies in his query are not used by the indexer, the retrieval fails.

To solve the vocabulary problem, a thesaurus is often used. A thesaurus contains a list of terms along with the relationships between them. During searching, a user can make use of the thesaurus to design the most appropriate search strategy. For example, if a search retrieves too few documents, a user can expand his query by consulting the thesaurus for similar terms. On the other hand, if a search retrieves too many documents, a user can use a more specific term suggested by the thesaurus. Manual construction of thesauri is a very complex 
process and often involves human experts. Previous research works have been done on automatic thesaurus construction [4].

In [6], Chen et. al. proposed the concept space approach to automatic thesaurus generation. A concept space is a network of terms and their weighted associations. The association between two terms is a quantity between 0 and 1 , computed from the co-occurrence of the terms from a given document collection. Its value represents the strength of similarity between the terms. When the association between two terms is zero, the terms have no similarity. It is because the terms never co-exist in a document. When the association from a term $a$ to another term $b$ is near to 1 , term $a$ is highly related to term $b$ in the document collection. Based on the idea of concept space, Schatz et al. constructed a prototype system to provide interactive term suggestion to searchers of the University of Illinois Digital Library Initiative test-bed [7]. Given a term, the system retrieves all the terms from a concept space that has non-zero associations to the given term. The associated terms are presented to the user in a list, sorted in decreasing order of association value. The user then selects new terms from the list to refine his queries interactively. Schatz showed that users could make use of the terms suggested by the concept space to improve the recall of their queries.

The construction of a concept space involves two phases: (1) an automatic indexing phase in which a document collection is processed to build inverted lists [4], and (2) a co-occurrence analysis phase in which the associations of every term pair are computed. Since there could be tens of thousands of terms in a document collection, computing the associations of all term pairs is very time consuming. In order to apply the concept space approach in large-scale document collections, efficient methods are needed.

We observe that in many applications, a complete concept space is not needed. In typical document collections, most of the associations are zero, i.e., most term pairs are not associated at all. Also, among the non-zero associations, only a very small fraction of them are having significant values. In typical applications, small-valued associations (or weak associations) are not useful. For example, in query augmentation, recommending weakly-associated terms to those keywords specified by a user query lowers the precision of the retrieval result.

In this paper, we propose and evaluate a number of efficient algorithms for constructing concept spaces that only contain strong associations. The challenge is how one could deduce that a certain term pair association is weak without actually computing it. We consider a number of pruning techniques that efficiently and effectively make such deductions.

The rest of the paper is organized as follows. In Section 2 we give a formal definition of concept space and discuss how term-pair associations are calculated. In Section 3 we consider three algorithms for the efficient construction of concept spaces that only contain strong associations. Experiment results comparing the performance of the algorithms are shown in Section 4. Finally, Section 5 concludes the paper. 


\section{Concept Space Construction}

A concept space contains the associations, $W_{j k}$ and $W_{k j}$, between any two terms $j$ and $k$ found in a document collection. Note that the associations are asymmetric, that is, $W_{j k} \neq W_{k j}$. According to Chen and Lynch [6], $W_{j k}$ is computed by the following formula:

$$
W_{j k}=\frac{\sum_{i=1}^{N} d_{i j k}}{\sum_{i=1}^{N} d_{i j}} \times \text { WeightingFactor }(k) .
$$

The symbol $d_{i j}$ represents the weight of term $j$ in document $i$ based on the term-frequency-inverse-document-frequent measure [2]:

$$
d_{i j}=t f_{i j} \times \log \left(\frac{N}{d f_{j}} \times w_{j}\right)
$$

where

$$
\begin{aligned}
t f_{i j} & =\text { number of occurrences of term } j \text { in document } i \\
d f_{j} & =\text { number of documents in which term } j \text { occurs } \\
w_{j} & =\text { number of words in term } j \\
N & =\text { number of documents. }
\end{aligned}
$$

The symbol $d_{i j k}$ represents the combined weight of both terms $j$ and $k$ in document $i$. It is defined as:

$$
d_{i j k}=t f_{i j k} \times \log \left(\frac{N}{d f_{j k}} \times w_{j}\right)
$$

where

$t f_{i j k}=$ number of occurrences of both terms $j$ and $k$ in document $i$, i.e., $\min \left(t f_{i j}, t f_{i k}\right)$, $d f_{j k}=$ number of documents in which both terms $j$ and $k$ occur.

Finally, WeightingFactor $(k)$ is defined as:

$$
\text { WeightingFactor }(k)=\frac{\log \left(N / d f_{k}\right)}{\log N} .
$$

WeightingFactor $(k)$ is used as a weighting scheme (similar to the concept of inverse document frequency) to penalize general terms (terms that appear in many documents). Terms with a high $d f_{k}$ value has a small weighting factor, which results in a small association value. Chen showed that this asymmetric similarity function $\left(W_{j k}\right)$ gives a better association than the popular cosine function [6]. Example 1 in the Appendix illustrates the computation of $W_{j k}$. 
In the following discussion, for simplicity, we assume that $w_{j}=1$ for all $j$ (i.e., all terms are single-word ones). We thus remove the term $w_{j}$ from the formula of $d_{i j}$ and $d_{i j k}$.

As we have mentioned, concept space construction is a two-phase process. In the first phase (automatic indexing), a term-document matrix, $T F$ is constructed. Given a document $i$ and a term $j$, the matrix $T F$ returns the term frequency, $t f_{i j}$. In practice, $T F$ is implemented using inverted lists. That is, for each term $j$, a linked list of [document-id,term-frequency] tuples is maintained. Each tuple records the occurrence frequency of term $j$ in the document with the corresponding id. Documents that do not contain the term $j$ are not included in the inverted list of $j$.

Besides the matrix, $T F$, the automatic indexing phase also calculates the quantity $d f_{j}$ (the number of documents containing term $j$ ) and well as $\sum_{i=1}^{N} t f_{i j}$ (the sum of the term frequency of term $j$ over the whole document collection) for each term $j$. These numbers are stored in arrays for fast retrieval during the second phase (co-occurrence analysis).

In the co-occurrence analysis phase, associations of every term pair are calculated. According to Equation 1 (page 3), to compute $W_{j k}$, we need to compute the values of three factors, namely, $\sum_{i=1}^{N} d_{i j k}, \sum_{i=1}^{N} d_{i j}$, and WeightingFactor $(k)$. Note that

$$
\sum_{i=1}^{N} d_{i j}=\sum_{i=1}^{N}\left[t f_{i j} \times \log \left(\frac{N}{d f_{j}}\right)\right]=\log \left(\frac{N}{d f_{j}}\right) \times \sum_{i=1}^{N} t f_{i j} .
$$

Since both $d f_{j}$ and $\sum_{i=1}^{N} t f_{i j}$ are already computed and stored during the automatic indexing phase, $\sum_{i=1}^{N} d_{i j}$ can be computed in constant time. Similarly, WeightingFactor $(k)$ can be computed in constant time as well.

Computing $\sum_{i=1}^{N} d_{i j k}$, however, requires much more work. From Equation 2, one needs to compute $d f_{j k}$ (i.e., the number of documents containing both terms $j$ and $k$ ) and $\sum_{i=1}^{N} t f_{i j k}$ in order to find $\sum_{i=1}^{N} d_{i j k}$. Figure 1 shows an algorithm for computing $W_{j k}$.

The execution time of Weight is dominated by the for-loop in line 3. Basically, most of the work is spent on scanning the inverted lists of terms $j$ and $k$.

\subsection{Algorithm A}

As we have mentioned, most of the associations are having zero or very small values. Our goal is to construct a concept space that contains only strong associations. Given a user-specified threshold $\lambda$, an association $W_{j k}$ is strong if $W_{j k} \geq \lambda$; otherwise, the association is weak.

To construct a concept space with only strong associations, our base algorithm first identifies all term pairs that have non-zero associations and then applies the function Weight on each pair. In particular, during the automatic indexing phase, a two-dimensional triangular bit matrix $C$ is built. The entry 


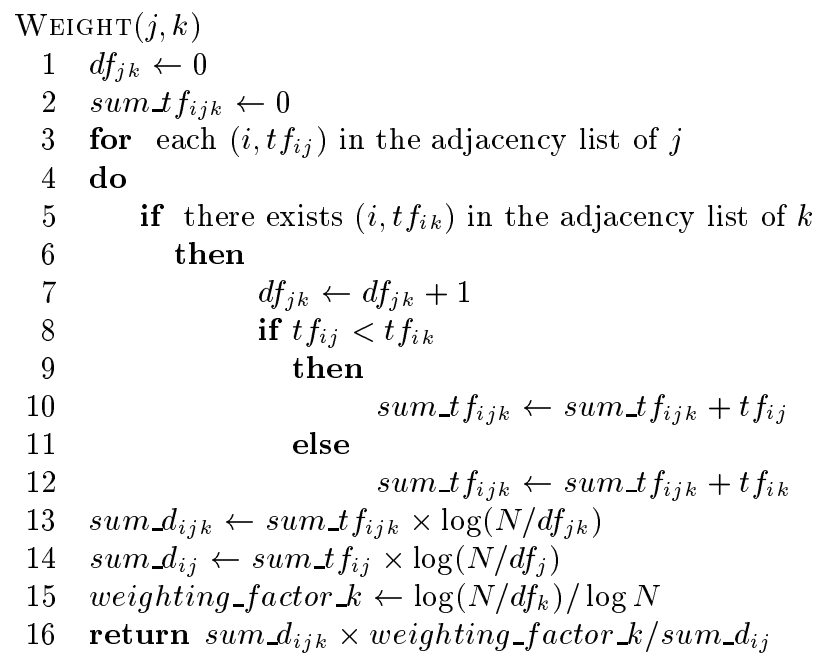

Fig. 1. Function Weight

$C(j, k)$ is set to 1 if there exists a document that contains both terms $j$ and $k$ (i.e., $\left.W_{j k}>0\right)$; otherwise, $C(j, k)$ is set to 0 .

During the co-occurrence analysis phase, the matrix $C$ is consulted. The associations, $W_{j k}$ and $W_{k j}$, are computed only if $C(j, k)$ is set. Associations that are less than $\lambda$ are discarded. We call this base Algorithm A(Figure 2). Example 2 in the Appendix shows how Algorithm A works.

\section{Pruning Algorithms}

Algorithm A is not particularly efficient. It basically computes all possible nonzero associations before filtering out those that are weak. As an example, we ran Algorithm A on a collection of 23,000 documents. It took 100 minutes for the algorithm to terminate. The major source of inefficiency lies in the Weight function, which scans two inverted lists for every non-zero association. In the collection, there are about 9 millions non-zero associations, and hence Algorithm A had to scan about 18 millions inverted lists.

In this section, we consider a few algorithms for improving the efficiency of Algorithm A. All of these algorithms share the following feature. Before computing an association $W_{j k}$, each one first computes an easy-to-compute estimate $W_{j k}^{\prime}$. If the estimate suggests that $W_{j k}$ is likely to be strong, the algorithm will execute the more expensive Weight function. The algorithms gain efficiency by avoiding the computation of weak associations. 


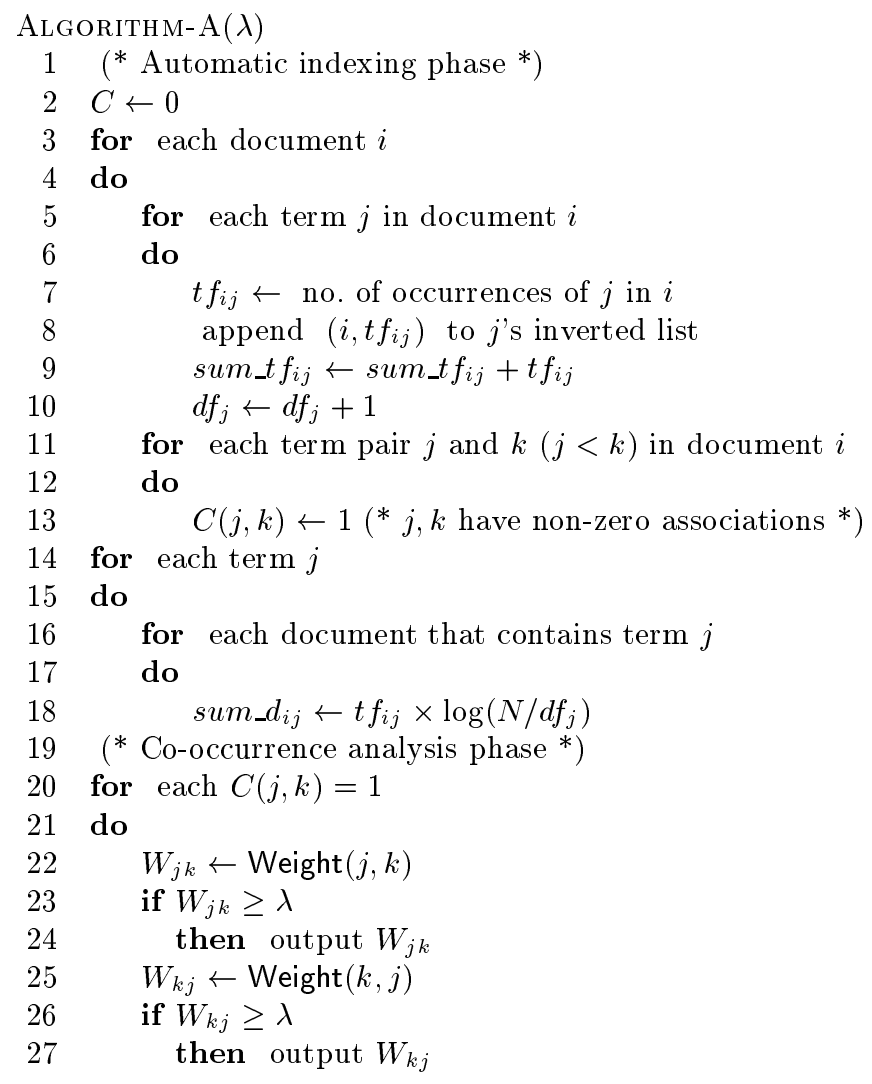

Fig. 2. Algorithm A 


\subsection{Algorithm B}

Our first efficient algorithm, B, computes an estimate $W_{j k}^{\prime}$ that is always an upper bound of $W_{j k}{ }^{1}$. If $W_{j k}^{\prime}$ is smaller than the threshold $\lambda$, we have $W_{j k} \leq W_{j k}^{\prime}<\lambda$. Hence, the association $W_{j k}$ must be weak and needs not be computed.

Recall that

$$
W_{j k}=\frac{\sum_{i=1}^{N} d_{i j k}}{\sum_{i=1}^{N} d_{i j}} \times \text { WeightingFactor }(k)
$$

and

$$
\sum_{i=1}^{N} d_{i j k}=\sum_{i=1}^{N}\left[t f_{i j k} \times \log \left(\frac{N}{d f_{j k}}\right)\right]=\log \left(\frac{N}{d f_{j k}}\right) \times \sum_{i=1}^{N} t f_{i j k} .
$$

By definition, $t f_{i j k}=\min \left(t f_{i j}, t f_{i k}\right)$. Note that

$$
\sum_{i=1}^{N} t f_{i j k}=\sum_{i=1}^{N} \min \left(t f_{i j}, t f_{i k}\right) \leq \min \left(\sum_{i=1}^{N} t f_{i j}, \sum_{i=1}^{N} t f_{i k}\right) .
$$

Also, unless the terms $j$ and $k$ are not negatively co-related, we have

$$
\frac{d f_{j k}}{N} \geq \frac{d f_{j}}{N} \times \frac{d f_{k}}{N}
$$

That is, the probability that a random document contains both terms $j$ and $k$ is at least as large as that probability when $j$ and $k$ are independent.

Now, consider $W_{j k}^{\prime}$, defined as:

$$
W_{j k}^{\prime}=\frac{\min \left(\sum_{i=1}^{N} t f_{i j}, \sum_{i=1}^{N} t f_{i k}\right) \times \log \left(\frac{N}{d f_{j}} \times \frac{N}{d f_{k}}\right)}{\sum_{i=1}^{N} d_{i j}} \times \text { WeightingFactor }(k) .
$$

We have $W_{j k}^{\prime} \geq W_{j k}$. Note that all the quantities that are needed to compute $W_{j k}^{\prime}$ are made available in the automatic indexing phase. Hence, $W_{j k}^{\prime}$ can be computed in constant time. Figure 3 shows the function Weight 1 for computing $W_{j k}^{\prime}$ and Figure 4 shows Algorithm B, which uses Weight1 as a pruning test.

\subsection{Algorithm C}

With $W_{j k}^{\prime}$, we replace the term $N / d f_{j k}$ by the bound $N^{2} /\left(d f_{j} \cdot d f_{k}\right)$. This bound can be very loose. Many weak associations $W_{j k}$ may have the estimate $W_{j k}^{\prime}$ exceeds $\lambda$ and hence the expensive Weight function is called. To improve the effectiveness of pruning, we consider another association estimate.

We first consider how the quantity $d f_{j k}$ can be estimated efficiently. For each term $j$, we compute a signature, $S_{j}$. Each signature is an array of $Q$ bits (with indices from 0 to $Q-1)$. Let $H()$ be a hash function such that, given a document

\footnotetext{
${ }^{1}$ This condition holds unless terms $j$ and $k$ are negatively co-related.
} 


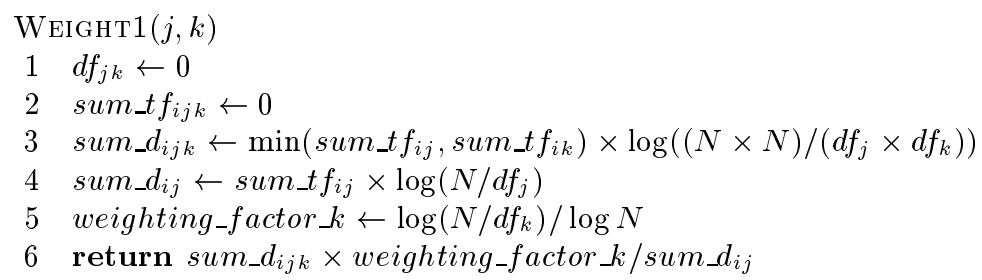

Fig. 3. Function Weight1

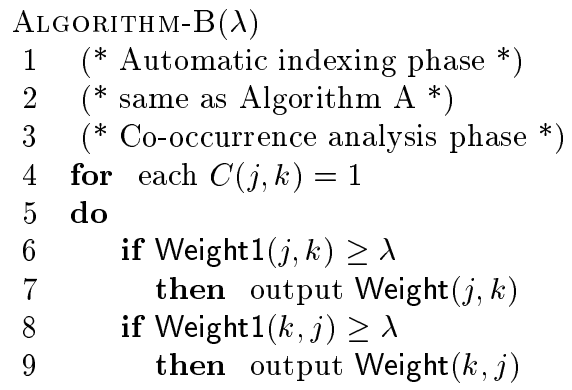

Fig. 4. Algorithm B

$i, H(i)$ returns an index in $[0 . . Q-1]$. For each document $i, S_{j}[H(i)]$ is set to 1 if document $i$ contains term $j$; otherwise $S_{j}[H(i)]$ is 0 .

Given two terms $j$ and $k$, we estimate $d f_{j k}$ by counting the number of ' 1 ' bits in the result of applying the bit-wise AND operation on the signatures $S_{j}$ and $S_{k}$. We denote this estimate by $\hat{d} f_{j k}$. Note that the estimate $\hat{d} f_{j k}$ could be incorrect if $Q$ is too small (which leads to many hash collisions). However, as we shall see in Section 4, setting $Q$ to $5 \%$ of the total number of documents in a collection is sufficient to reduce the error probability to a negligible level. Also, we note that computing $\hat{d} f_{j k}$ using the small bit-vector signatures is much faster than computing the exact value of $d f_{j k}$ by scanning the inverted lists.

Another estimate we consider is to approximate the value $\sum_{i=1}^{N} t f_{i j k}$. First, if we use $\max _{p}\left(t f_{p j}\right)$ to denote the largest term frequency of a term $j$ over any document, then clearly, $t f_{i j} \leq \max _{p}\left(t f_{p j}\right)$ for any document $i$. Hence,

$$
t f_{i j k}=\min \left(t f_{i j}, t f_{i k}\right) \leq \min \left(\max _{p}\left(t f_{p j}\right), \max _{p}\left(t f_{p k}\right)\right) .
$$

Thus,

$$
\begin{aligned}
\sum_{i=1}^{N} t f_{i j k} & =\sum_{j, k \in \operatorname{doc} i} t f_{i j k} \leq \sum_{j, k \in \operatorname{doc} i} \min \left(\max _{p}\left(t f_{i j}\right), \max _{p}\left(t f_{i k}\right)\right) \\
& =d f_{j k} \times \min \left(\max _{p}\left(t f_{p j}\right), \max _{p}\left(t f_{p k}\right)\right)
\end{aligned}
$$


Substituting these estimates to the association formula, we obtain

$$
W_{j k}^{\prime \prime}=\frac{\min \left(\max \left(t f_{i j}\right), \max \left(t f_{i k}\right)\right) \times \hat{d} f_{j k} \times \log \left(\frac{N}{\hat{d} f_{j k}}\right)}{\sum_{i=1}^{N} d_{i j}} \times \text { WeightingFactor }(k) .
$$

Note that $W_{j k}^{\prime \prime}$ can be computed in constant time. Example 3 in the Appendix illustrates how $W_{j k}^{\prime \prime}$ is computed.

Figure 5 shows the function Weight2 for computing $W_{j k}^{\prime \prime}$. Comparing Weight1 and Weight2, we note that Weight1 is a bit more efficient than Weight2 since no bit vector processing is needed. However, the estimate computed by Weight 1 is less tight. Our algorithm (Algorithm C) thus uses Weight1 as the first pruning test, then applies Weight2 if necessary. Figure 6 shows Algorithm C.

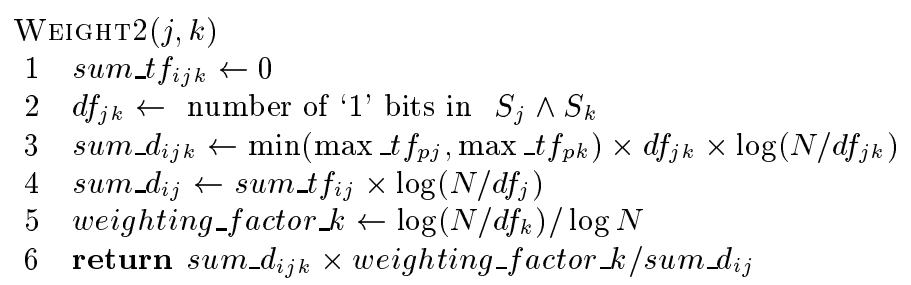

Fig. 5. Function Weight2

\subsection{Algorithm D}

In Algorithm C, the quantity $d f_{j k}$ is estimated by processing the signatures $S_{j}$ and $S_{k}$. We note that $d f_{j k}$ appears only in a log term (see Equation 2). A mild mis-estimation of $d f_{j k}$ should not affect the value of the association $W_{j k}$ by much. Moreover, by inspecting a few document collections, we discovered that for terms that have a not-too-small association (e.g., > 0.6), it is almost always the case that $d f_{j k}=\min \left(d f_{j}, d f_{k}\right)$. Our next algorithm uses this observation to compute a quick estimate, $W_{j k}^{\prime \prime \prime}$, for the association between terms $j$ and $k$ :

$$
W_{j k}^{\prime \prime \prime}=\frac{\min \left(\sum_{i=1}^{N} t f_{i j}, \sum_{i=1}^{N} t f_{i k}\right) \times \log \left(\frac{N}{\min \left(d f_{j}, d f_{k}\right)}\right)}{\sum_{i=1}^{N} d_{i j}} \times \text { WeightingFactor }(k) .
$$

Same as Weight1, we estimate $\sum_{i=1}^{N} t f_{i j k}$ by an upper bound $\min \left(\sum_{i=1}^{N} t f_{i j}, \sum_{i=1}^{N} t f_{i k}\right)$. Also, we use $\min \left(d f_{j}, d f_{k}\right)$ to approximate $d f_{j k}$. Figure 7 shows the function Weight3 for computing $W_{j k}^{\prime \prime \prime}$. Figure 8 shows Algorithm D which uses Weight3 as the pruning test. 


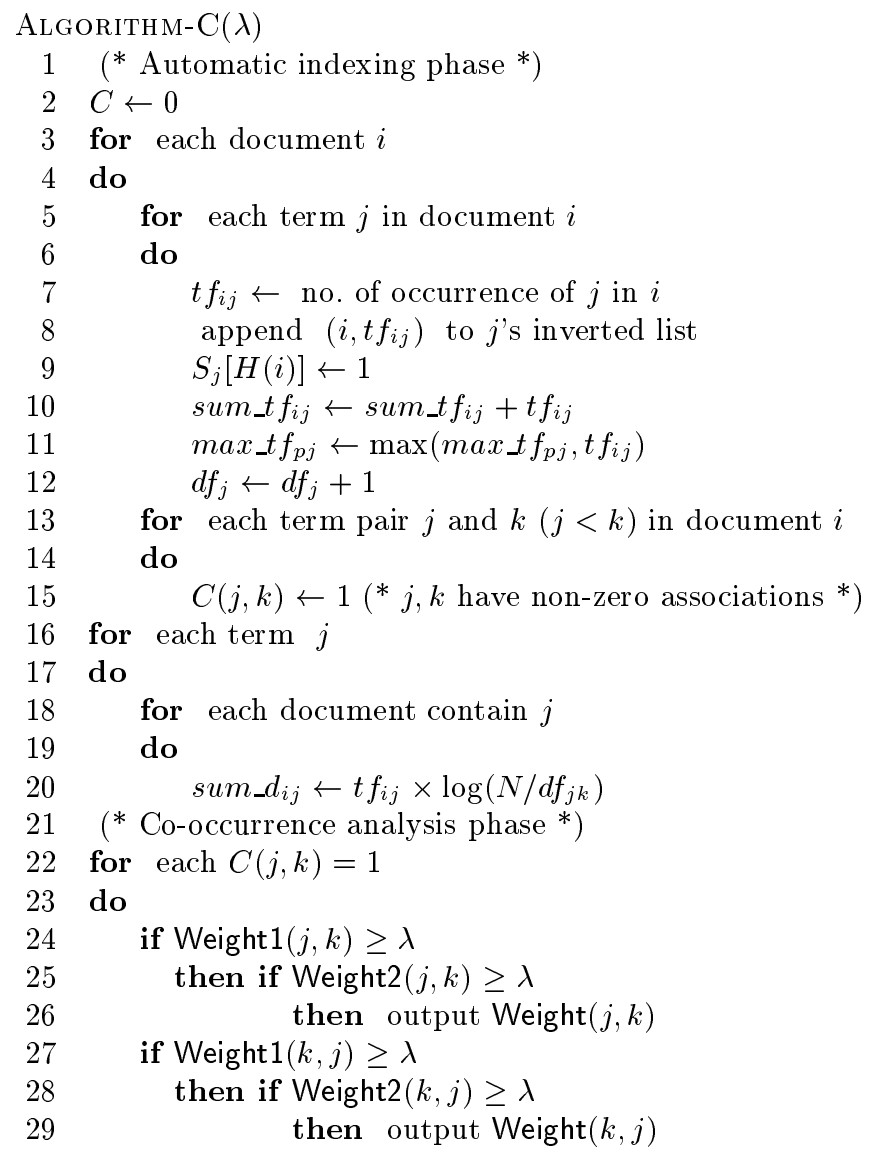

Fig. 6. Algorithm C

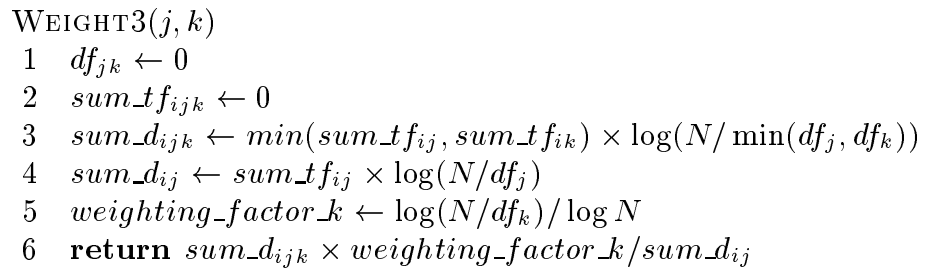

Fig. 7. Function Weight3 


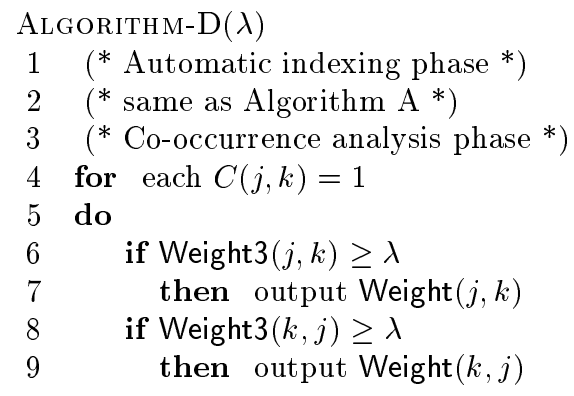

Fig. 8. Algorithm D

\section{Performance Evaluation}

In this section we compare the performance of the four algorithms. Due to space limitation, only some representative results are shown.

We applied the algorithms on a few document collections. For example, we had a medical document collection taken from the Smart project [1], and a collection of web pages taken from a news web site. For illustration, we show the performance results using the collection of news documents.

The document collection consists of 22,613 documents with 55,772 terms. The document database is 20.9 MBytes large (after stop-word removal and stemming). There are about $1.2 \times 10^{8}$ non-zero associations in the dataset. The bar graph in Figure 9 shows the frequency distributions of the non-zero associations under 10 ranges of association values. This distribution shows that only a very small fraction of the associations have large values.

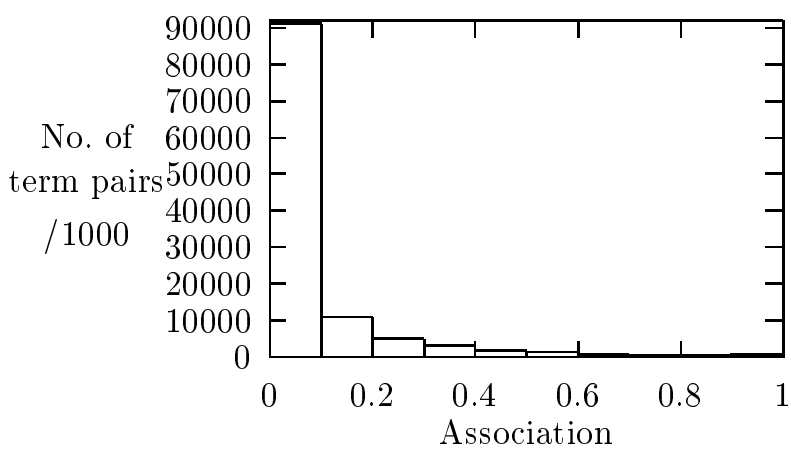

Fig. 9. Distribution of non-zero associations 
We ran the algorithms A, B, C, and D on a $250 \mathrm{MHz}$ UltraSparc machine. Figure 10 shows the runtime of the algorithms. For Algorithm B, the number of bits for a signature vector is set to $5 \%$ of the total number of documents in the collection (i.e., 2,789 bits).

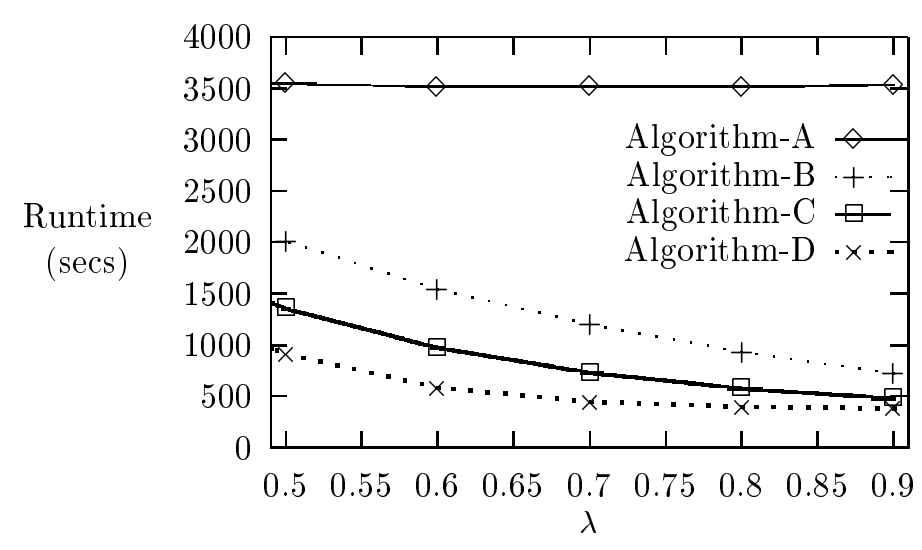

Fig. 10. Runtime (secs) of algorithms

From the figure, we see that Algorithm $\mathrm{A}$ is the slowest, taking about 3,500 seconds to complete. The runtime of Algorithm $\mathrm{A}$ is independent of $\lambda$, since it simply computes all non-zero associations. Algorithms B, C, and D are much faster than A. For example, when $\lambda=0.9$, Algorithm D only takes 380 seconds a speedup of 9.2 times over Algorithm A.

Among the three pruning algorithms, Algorithm D is the most efficient, followed by $\mathrm{C}$, and then by B. Moreover, their speed increases with $\lambda$. This is because a larger $\lambda$ value means that more associations are considered weak. This allows the algorithms to prune more weak associations.

It is interesting to see how much unnecessary work that each algorithm has done. Figure 11 shows the number of weak associations that each algorithm has computed (using the Weight function). The figure shows, for example, that when $\lambda=0.9$, Algorithm B computed about 20 million associations whose values are less than 0.9. Algorithm A, on the other hand, computed about 110 million of such associations, or 5.5 times as many as that of Algorithm B. Algorithms C and $\mathrm{D}$ are even more effective in pruning weak associations. For example, at $\lambda=0.9$, Algorithm D only computed 349638 weak associations, or $3 \%$ of that of Algorithm A. Finally, we note that even though algorithms C and D are equally effective in pruning weak associations, Algorithm $C$ is less efficient than Algorithm D. This is because Algorithm C uses signature vectors $S_{j}$ and $S_{k}$ to estimate $d f_{j k}$. Processing these signatures requires $O(Q)$ time, where $Q$ is the number of bits in a signature vector. Computing the function Weight2 thus takes $O(Q)$ time. 
Algorithm D, on the other hand, computes the function Weight3 in constant time. Hence, Algorithm D is faster.

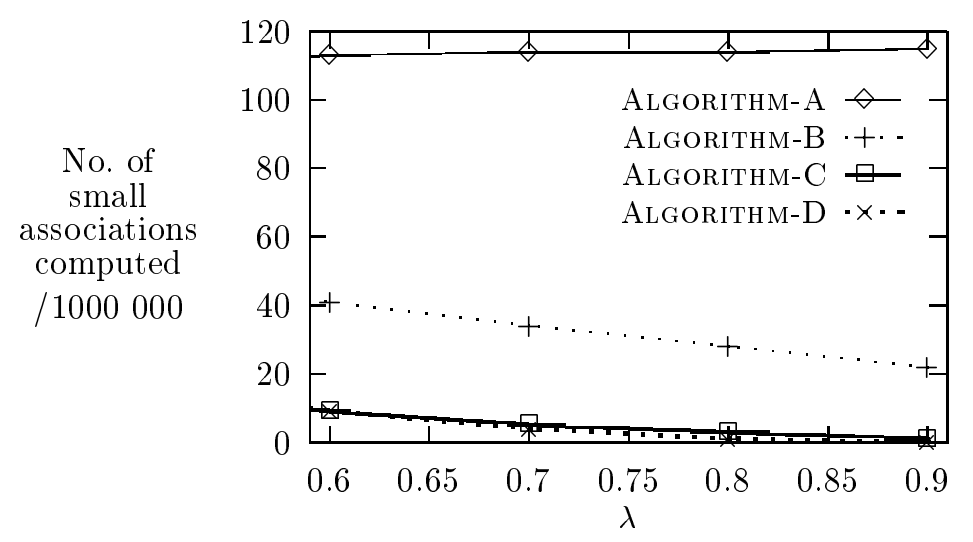

Fig. 11. Number of weak associations computed by the algorithms

As we have discussed, the efficiency of the pruning algorithms come from the use of estimates that act as pruning tests to filter out weak associations (without computing them). Although very unlikely, it is possible that these pruning tests fail. That is to say, it is possible (with a very small probability) that a strong association is mistakenly indicated by a pruning test to be weak. In such a case, the association is (erroneously) not computed. We compare the output of Algorithm $A$ with those of the three pruning algorithms. Fortunately, we discover that only a very small fraction of the strong associations are discarded. In particular, Algorithm B never misses any strong associations; also, for $\lambda \geq 0.7$, algorithms $\mathrm{C}$ and D missed at most 8 and 7 strong associations (out of 1844552).

\section{Conclusion}

This paper studied the problem of concept space construction. Previous studies have shown that the concept space approach to automatic thesaurus construction is a useful tool for information retrieval. The construction of concept spaces, however, is very time consuming. In many applications, a full concept space is not needed, in particular, only strong associations are used. We proposed three pruning algorithms for constructing concept spaces containing only strong associations. We evaluated these algorithms using a number of document collections. We found that the three pruning algorithms are very effective in avoiding the computation of unneeded weak associations. A 10-time speedup of the construction process can be achieved. 


\section{References}

1. The Smart Project. ftp://ftp.cs.cornell.edu/pub/smart/med/.

2. R. Baeza-Yates and Berthier Ribeiro-Neto. Modern Information Retrieval. Addison Wesley, 1999.

3. Hsinchun Chen, Joanne Martinez, Tobun D. Ng, and Bruce R. Schatz. A concept space approach to addressing the vocabulary problem in scientific information retrieval: an experiment on the worm community system. Journal of American Society for information Science, 48(1):17-31, 1997.

4. W.B. Frakes and R. Baeza-Yates. Information Retreival: Data Structures and Algorithms. Prentice Hall, 1992.

5. G.W. Furnas et al. The vocabulary problem in human-system communicaiton. Comm. ACM, 30(11):964-971, 1987.

6. H.Chen and K.J. Lynch. Automatic construction of networks of concepts characterizing document databases. IEEE Transaction of Systems, Man, and Cybernetics, 22(5):885-902, Sep/Oct 1992.

7. B.R. Schatz, E. Johnson, P. Cochrane, and H. Chen. Interactive term suggestion for users of digital libraries: using subject thesauri and co-occurrence lists for information retrieval. In Digital Library 96, Bethesda MD, 1996. 


\section{Appendix}

Example 1. Given a document collection containing 6 documents $D_{1} \ldots D_{6}$, Table 1 shows the term-doc matrix, $T F$, which records the number of occurrences of each term $(j, k, l, m, n, o, p, q)$ in each document. For instance, the first entry of the first row gives the value of $t f_{1 j}$.

\begin{tabular}{|c|c|c|c|c|c|c|}
\hline \multirow{2}{*}{ Terms } & \multicolumn{7}{|c|}{ Documents } \\
\cline { 2 - 7 } & $D_{1}$ & $D_{2}$ & $D_{3}$ & $D_{4}$ & $D_{5}$ & $D_{6}$ \\
\hline$j$ & 0 & 5 & 0 & 0 & 0 & 4 \\
\hline$k$ & 0 & 4 & 0 & 0 & 1 & 1 \\
\hline$l$ & 2 & 0 & 3 & 0 & 0 & 0 \\
\hline$m$ & 5 & 0 & 2 & 0 & 0 & 0 \\
\hline$n$ & 1 & 0 & 0 & 4 & 2 & 0 \\
\hline$o$ & 0 & 0 & 0 & 1 & 3 & 0 \\
\hline$p$ & 0 & 3 & 0 & 0 & 0 & 3 \\
\hline$q$ & 0 & 0 & 5 & 0 & 4 & 0 \\
\hline
\end{tabular}

Table 1. An example $T F$

Recall that the association from a term $j$ to a term $k, W_{j k}$, is defined as:

$$
W_{j k}=\frac{\sum_{i=1}^{N} d_{i j k}}{\sum_{i=1}^{N} d_{i j}} \times \text { WeightingFactor }(k) .
$$

To compute the association, we first compute the quantity $\sum_{i=1}^{N} d_{i j k}$. Recall that $d_{i j k}=t f_{i j k} \times \log \left(\frac{N}{d f_{j k}} \times w_{j}\right)$. The term $t f_{i j k}$ is the number of occurrences of terms $j$ and $k$ in document $i$. This value can be obtained by scanning through the rows in $T F$ that are labelled $j$ and $k$, and taking the smaller value of $t f_{i j}$ and $t f_{i k}$ for each $i$.

From the matrix in Table $1, t f_{1 j k}=0, t f_{2 j k}=4, t f_{3 j k}=0, t f_{4 j k}=$ $0, t f_{5 j k}=0, t f_{6 j k}=1$. Similarly, $d f_{j k}$ can be obtained by scanning through the rows in $T F$ labelled $j$ and $k$, and counting the number of documents that contain both $j$ and $k$. In the example, $d f_{j k}$ is 2 . In our example, we assume that all terms are of single word. Hence, all $w_{j}$ 's are 1 . It follows that,

$$
\sum_{i=1}^{6} d_{i j k}=\sum_{i=1}^{6}\left[t f_{i j k} \times \log \left(\frac{6}{d f_{j k}}\right)\right]=(0+4+0+0+0+1) \times \log \frac{6}{2}=5.493
$$

To compute the quantity $\sum d_{i j}$ for each document $i$, recall that $d_{i j}=t f_{i j} \times$ $\log \left(\frac{N}{d f_{j}} \times w_{j}\right)$. The quantity $d f_{j}$ can be obtained by counting the number of nonzero entries in the row labelled $j$. In this case, $d f_{j}$ is 2 . Again, $w_{j}$ is 1 . It follows that, 


$$
\sum_{i=1}^{6} d_{i j}=\sum_{i=1}^{6}\left[t f_{i j} \times \log \left(\frac{6}{d f_{j}}\right)\right]=(0+5+0+0+0+4) \times \log \left(\frac{6}{2}\right)=9.888
$$

Finally, the weighting factor WeightingFactor $(k)$ is defined as $\log \frac{N}{d f_{k}} / \log N$. For our example, $N$ is 6 and $d f_{k}$ is 3 . Therefore,

$$
\text { WeightingFactor }(k)=\frac{\log \left(\frac{6}{3}\right)}{\log 6}=0.387
$$

It follows that, $W_{j k}=(5.493 \times 0.386) / 9.888=0.214$.

Example 2. Consider the document collection in Example 1. Algorithm A starts by initializing $C$ as a bit matrix containing zero in all of its entries. It then scans each of the documents one by one to find term pairs that co-exist in one or more documents. For example, the algorithm finds that in document $D_{1}$ the terms $l, m$ and $n$ co-exist. It thus sets $C(l, m), C(l, n)$ and $C(m, n)$ to 1 . After the algorithm has scanned all the six documents, the bit matrix $C$ becomes:

\begin{tabular}{|l|l|l|l|l|l|l|l|l|}
\hline & $j$ & $k$ & $l$ & $m$ & $n$ & $o$ & $p$ & $q$ \\
\hline$j$ & 0 & 1 & 0 & 0 & 0 & 0 & 1 & 0 \\
\hline$k$ & 0 & 0 & 0 & 0 & 1 & 1 & 1 & 1 \\
\hline$l$ & 0 & 0 & 0 & 1 & 1 & 0 & 0 & 1 \\
\hline$m$ & 0 & 0 & 0 & 0 & 1 & 0 & 0 & 1 \\
\hline$n$ & 0 & 0 & 0 & 0 & 0 & 1 & 0 & 1 \\
\hline$o$ & 0 & 0 & 0 & 0 & 0 & 0 & 0 & 1 \\
\hline$p$ & 0 & 0 & 0 & 0 & 0 & 0 & 0 & 0 \\
\hline$q$ & 0 & 0 & 0 & 0 & 0 & 0 & 0 & 0 \\
\hline
\end{tabular}

Each non-zero entry in the matrix $C$ indicates the existence of a non-zero association between two terms. For instance, since $C(j, k)=1$, Algorithm A computes both $W_{j k}$ and $W_{k j}$. Every term pair having a value 1 in the $C$ matrix is processed similarly, and the algorithm outputs all associations of value not less than $\lambda$.

Example 3. Consider the document collection in Example 1. Assume $Q=3$ and $H(i)=(i \bmod Q)$. The estimated value of $W_{j k}$ is computed as follows. First, from the $j$-th row and the $k$-th row of the term-doc matrix $T F$ we compute the corresponding $S_{j}$ and $S_{k}$ vectors:

$$
\begin{gathered}
\begin{array}{|c||c|c|c|c|c|c|}
\hline & D_{1} & D_{2} & D_{3} & D_{4} & D_{5} & D_{6} \\
\hline j & 0 & 5 & 0 & 0 & 0 & 4 \\
\hline
\end{array} \\
\begin{array}{|c||c|c|c|c|c|c|}
\hline & D_{1} & D_{2} & D_{3} & D_{4} & D_{5} & D_{6} \\
\hline k & 0 & 4 & 0 & 0 & 1 & 1 \\
\hline
\end{array}
\end{gathered}
$$


Here, $S_{j}=\{1,0,1\}$ and $S_{k}=\{1,0,1\}$. Since both $S_{j}$ and $S_{k}$ have a ' 1 ' bit at positions 0 and 2 , the estimated value of $d f_{j k}$ is 2 . From $T F, \max _{i}\left(t f_{i j}\right)=5$ and $\max _{i}\left(t f_{i k}\right)=4$. The value of $W_{j k}^{\prime \prime}$ is:

$$
\begin{aligned}
W_{j k}^{\prime \prime} & =\frac{\min \left(\max \left(t f_{i j}\right), \max \left(t f_{i k}\right)\right) \times \hat{d} f_{j k} \times \log \left(\frac{N}{\hat{d} f_{j k}}\right)}{\sum_{i=1}^{N} d_{i j}} \times \text { WeightingFactor }(k) \\
& =\frac{\min \left(\max \left(t f_{i j}\right), \max \left(t f_{i k}\right)\right) \times \hat{d} f_{j k} \times \log \left(\frac{N}{d f_{j k}}\right)}{\sum_{i=1}^{N} t f_{i j} \times \log \left(\frac{N}{d f_{j}}\right)} \times \frac{\log \frac{N}{d f_{k}}}{\log N} \\
& =\frac{\min (5,4) \times 2 \times \log \left(\frac{6}{2}\right)}{9 \times \log \left(\frac{6}{2}\right)} \times \frac{\log \frac{6}{3}}{\log 6} \\
& =0.344
\end{aligned}
$$

